Draft Version August 15, 2018

Typeset using IATEX RNAAS style in AASTeX62

\title{
SPECTRAL CURVATURE OF SHOCK-ACCELERATED PARTICLES IN SOLAR CYCLE 23
}

\author{
Connie Zhou, ${ }^{1}$ Federico Fraschetti, ${ }^{2}$ Jeremy J. Drake, ${ }^{3}$ and Martin Pohl ${ }^{4}$ \\ ${ }^{1}$ Harvard/Smithsonian Center for Astrophysics, 60 Garden St., Cambridge, MA, USA, 02138; Winsor School, Boston, MA, USA, 02215 \\ ${ }^{2}$ Harvard/Smithsonian Center for Astrophysics, 60 Garden St., Cambridge, MA, USA, 02138; Depts. of Planetary Sciences and \\ Astronomy, Tucson, AZ, USA, 85721 \\ ${ }^{3}$ Harvard/Smithsonian Center for Astrophysics, 60 Garden St., Cambridge, MA, USA, 02138 \\ ${ }^{4}$ DESY, 15738 Zeuthen, Germany; Institute of Physics and Astronomy, University of Potsdam, 14476 Potsdam, Germany
}

Keywords: editorials, notices — miscellaneous — catalogs — surveys

In the late '70s, the acceleration of charged particles at astrophysical shock waves was theoretically predicted, within the diffusive shock acceleration model, to result in a power-law momentum distribution of the accelerated particles, i.e., $N(\gamma)=N_{0}\left(\gamma / \gamma_{0}\right)^{-\alpha}$, where $\gamma$ is the electrons Lorentz factor and $\alpha$ the power law index (Bell 1978; Blandford \& Ostriker 1978). Recently, Fraschetti \& Pohl (2017a) modelled the baseline photon spectrum of the Crab Nebula, the remnant of a supernova explosion that occurred in AD 1054 and showed that a single log-parabola spectrum of energetic electrons, accelerated at the termination shock of the Nebula, can reproduce the observations over 21 orders of magnitude in photon energy. The log-parabola takes the form $N(\gamma)=N_{0}\left(\gamma / \gamma_{0}\right)^{-\alpha-\beta \log \left(\gamma / \gamma_{0}\right)}$, where $\beta$ is the spectral curvature.

Double power-laws in solar energetic particle (hereafter SEPs) spectra have been observed for more than a decade (e.g. Mason et al. 2002; Mewaldt et al. 2005). Li \& Lee (2015) have shown that the Parker equation with power-law injected particles near the Sun admits a double power-law as a solution at $1 \mathrm{AU}$ if one includes an energy dependence of the scattering mean free path and adiabatic deceleration in the radially divergent solar wind. However, that study neglects perpendicular diffusion (e.g. Fraschetti \& Jokipii 2011) that plays a relevant role in large gradual SEP events (Dröge et al. 2016, and references therein). Mewaldt et al. (2012) reported the proton spectra of the 16 Ground Level Events (hereafter GLEs), i.e., SEPs events with a proton flux exceeding the background of galactic cosmic rays in the neutron monitors (Lopate 2006), that occurred during solar cycle 23. The spectra of such possibly shock-accelerated protons (in the range $\sim 0.1 \mathrm{MeV}$ to $500-700 \mathrm{MeV}$ ) can be reproduced (Mewaldt et al. 2012) by the Band function (Band et al. 1993). This is an empirical fitting function originally introduced to fit the $\gamma$-ray spectra of Gamma Ray Bursts, and comprises two power-laws with different index continuously joined at an energy break $E_{0}$.

In this study, we have used the GLE spectra of solar cycle 23 to establish a relation of $\beta$ to $E_{0}$. We assume that the measured spectra do not change since the injection, likely to happen in shocks driven by gradual coronal mass ejections. The log-parabola spectrum results from the leakage of the highest-energy particles that leads to the spectral softening and is described in terms of the probability of containing such particles in proximity of the shock (Massaro et al. 2004; Fraschetti \& Pohl 2017a,b). As the particle energy grows, the non-planarity of the shock surface and the greater scattering mean free path modify this probability by introducing an energy-dependence.

We have used multi-spacecraft 1-day integrated proton spectra of the GLEs of solar cycle 23 from ACE/ULEIS, ACE/EPAM, GOES-8/11, SAMPEX-PET instruments, available at the energetic particles repository http: //www.srl.caltech.edu/sampex/ DataCenter/DATA/EventSpectra/index_ace.html. We have carried out a best-fit of the entire GLE sample. Figure 1, left panel, shows the log-parabola best-fit of the August $24^{\text {th }} 2002$ event in comparison with the two asymptotic power-laws from the Band function best-fit. Figure 1, right panel, shows for each of the 16 events our best-fit $\beta$ vs the best-fit $E_{0}$ from Mewaldt et al. (2012). Despite the limited sample available, we find an anticorrelation of the best-fit parameters. The only event with $E_{0}>100 \mathrm{MeV}$ has a large relative error for both $\beta(41 \%)$ and $E_{0}(28 \%)$ due to the little spectral curvature in the energy range considered, i.e., $0.08-100 \mathrm{MeV}$. 
The two events with $E_{0}<5 \mathrm{MeV}$ have a spectral coverage $\leq 3$ decades with also a resulting poorer determination of $E_{0}($ and $\beta)$.

In summary, our finding shows for most of the 16 GLE events of cycle 23 an anti-correlation $\beta \simeq E_{0}^{-0.36}$. Such an anti-correlation results from the fact that a shock structure confining higher-energy particles (higher $E_{0}$ ) enables particle acceleration via the diffusive mechanism up to higher energies with a spectrum closer to a power-law (smaller $\beta$ ). A log-parabola fit allows also for a reduced number of free parameters, modulo a normalization: one power-law index $(\alpha)$ and a global spectral curvature $(\beta)$ as opposed to two power-laws indexes and the break $E_{0}$. Thus, we propose to replace the single energy value of the break $E_{0}$ with the parameter $\beta$ that describes a global spectral behaviour.
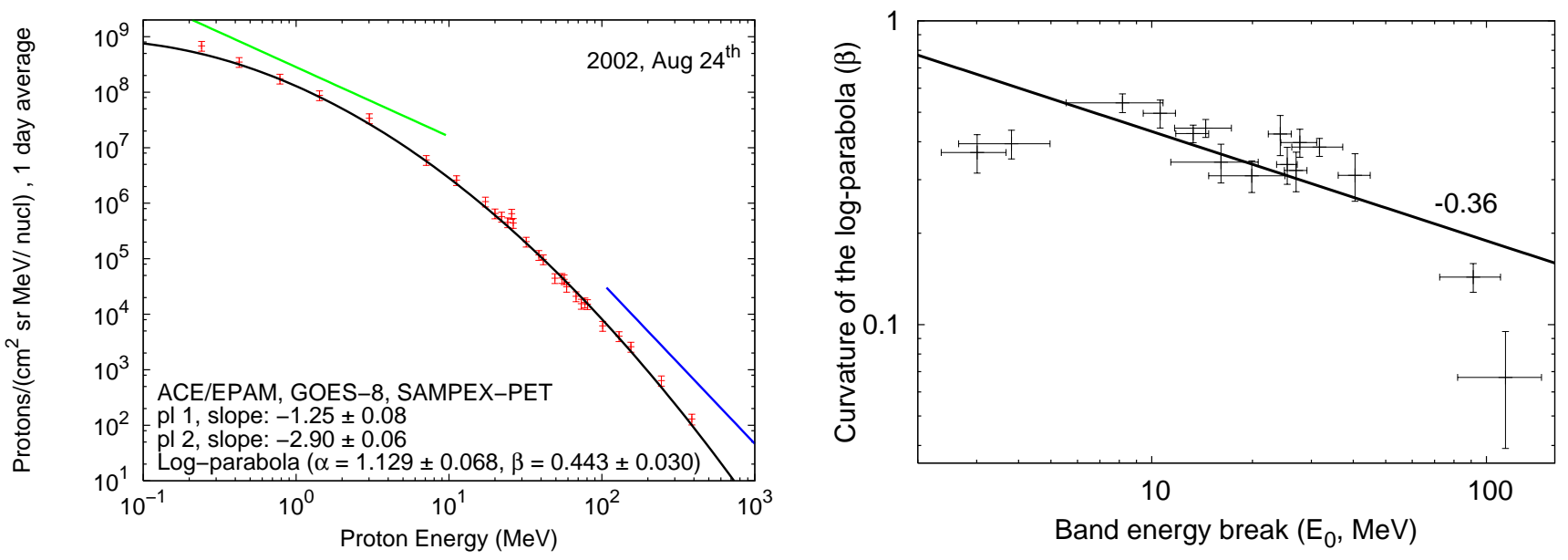

Figure 1. Left: Fluence 1-day integrated spectrum as measured by ACE/EPAM, GOES-8 and SAMPEX-PET during the event on August $24^{\text {th }} 2002$ is fitted with a log-parabola spectrum; asymptotic power law indexes from Band function best-fit (Mewaldt et al. 2012) are reported. Right: For all 16 GLE events in solar cycle 23, best-fit curvature of the log-parabola vs best-fit energy break of the Band function.

We are grateful for the assistance of Dr. R. Mewaldt on multi-spacecraft data and for reading the manuscript. CZ thanks Harvard University for the hospitality and Winsor School ILE program. FF acknowledges support from NASA under Grant NNX15AJ71G and Scholarly Studies Award 40488100HH00181 at Harvard/Smithsonian Center for Astrophysics. 


\section{REFERENCES}

Band, D., Matteson, J., Ford, L., et al. 1993, ApJ, 413, 281

Bell, A. R. 1978, MNRAS, 182, 147

Blandford, R. D., \& Ostriker, J. P. 1978, ApJL, 221, L29

Dröge, W., Kartavykh, Y. Y., Dresing, N., \& Klassen, A. 2016, ApJ, 826, 134

Fraschetti, F., \& Jokipii, J. R. 2011, ApJ, 734, 83

Fraschetti, F., \& Pohl, M. 2017a, MNRAS, 471, 4856

Fraschetti, F., \& Pohl, M. 2017b, in European Physical Journal Web of Conferences, Vol. 136, 02009

Li, G., \& Lee, M. A. 2015, ApJ, 810, 82
Lopate, C. 2006, Fifty Years of Ground Level Solar Particle Event Observations, Washington DC American Geophysical Union Geophysical Monograph Series, 165, 283

Mason, G. M., Wiedenbeck, M. E., Miller, J. A., et al. 2002, ApJ, 574, 1039

Massaro, E., Perri, M., Giommi, P., \& Nesci, R. 2004, $A \& A, 413,489$

Mewaldt, R. A., Cohen, C. M. S., Labrador, A. W., et al. 2005, Journal of Geophysical Research (Space Physics), 110, A09S18

Mewaldt, R. A., Looper, M. D., Cohen, C. M. S., et al. 2012, SSRv, 171, 97 